\title{
Experimental evidence of a fundamental noise floor at the tens of millihertz level in laser locking onto unbalanced fibre-based Michelson interferometer
}

\author{
Frédéric Audo, Jean-Pierre Coulon, Fabien Kéfélian \\ Laboratoire ARTEMIS, Observatoire de la Côte d'Azur, Université Côte d'Azur, CNRS, Bvd de l'Observatoire 06304 Nice, France
}

Large arm length imbalance fibre-based interferometers have shown great potential for laser frequency stabilization and control, with frequency noise power spectral density close to the $10^{-1} \mathrm{~Hz} / \mathrm{Hz}^{1 / 2}$ level [1]. In order to understand the performance of such frequency stabilization systems, it is important to distinguish the intrinsic noise of the frequency reference, which is ultimately limited by fibre thermal noise, and the locking noise, which is limited by the detection noise but can also be affected by residual amplitude modulation fluctuations in phase modulator or other less well known causes. It is often difficult to measure the out-of-loop locking noise when one laser is locked to a frequency reference. However, the fibre length noise practically cancels when two lasers are locked onto the same interferometer with a small frequency difference, which allows to measure the out-of-loop locking noise to a very low level [2].

Fig. 1 (a) shows our experimental setup. Two low frequency noise diode lasers at 1,5 $\mu \mathrm{m}$ from RIO, with frequency difference in the microwave range, are independently locked on the same Michelson interferometer with a $300 \mathrm{~m}$ fibre spool in one arm, using the Pound-Drever-Hall (PDH) technique. The two PDH RF signals are detected by the same photodiode followed by a shot noise limited home-made transimpedance amplifier. The photodetector signal is split, filtered and down-mixed to generate two error signals which are amplified by a servo circuit with 4 integrators in order to reach very low residual laser noise at $1 \mathrm{kHz}$ despite a bandwidth below $100 \mathrm{kHz}$. Frequency corrections are applied through the diode current and the electro-optic voltage. The beat-note frequency noise spectrum was measured by using a home-made frequency-to-voltage convertor. Fig. 1 (b) shows the spectrum of the frequency noise between the free running (dark) and the locked lasers (blue), the frequency noise converted from the error signals (red) and the measurement floor (grey). We clearly observe on the spectrum of the frequency noise between the two locked lasers, i.e. the out-of-loop locking noise, a white floor of $2 \times 10^{-1} \mathrm{~Hz} / \mathrm{Hz}^{1 / 2}$, largely above the in-loop error noise floor.

(a)

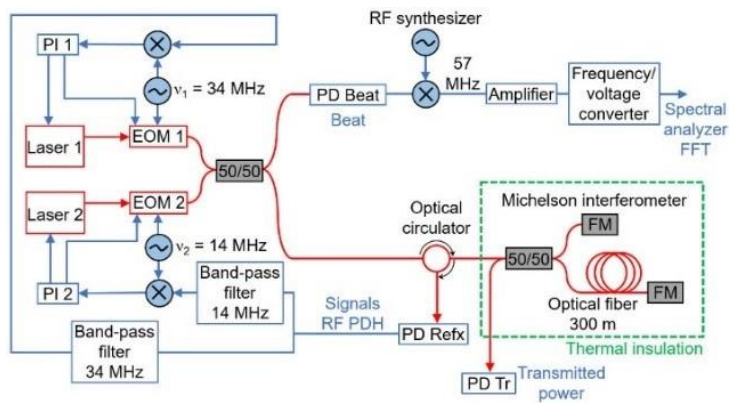

(b)

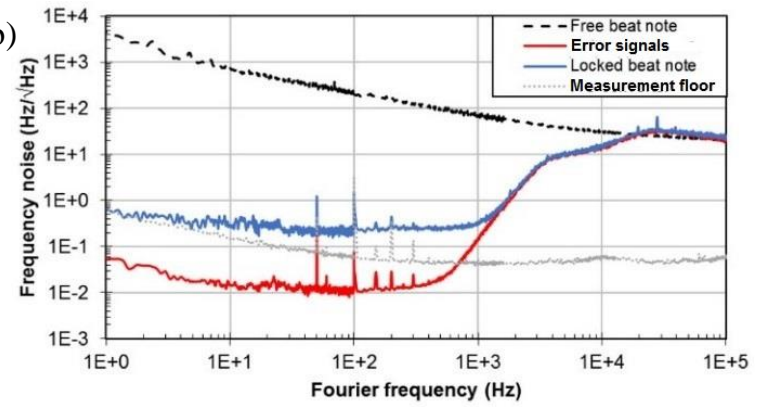

Fig. 1 (a) Scheme of the experiment, EOM Electro-Optic Modulator, FM Faraday Mirror, PI proportional-integrator, PD Photodetector. (b) Frequency noise spectra between the two lasers, in loop error noise floor and measurement floor

Multiple tests performed on the setup have shown that this noise floor is independent of the frequency difference between the two lasers, independent of the detection power level, on the way correction was applied and also on different interferometer configurations and was not due to any cross-talk or multimode effects. However using two fibre lasers with a frequency noise lower than the RIO laser, the noise floor have decreased to $9 \times 10^{-2} \mathrm{~Hz} / \mathrm{Hz}^{1 / 2}$ (corresponding to a Lorentzian linewidth of $25 \mathrm{mHz}$ ). Moreover, adding a single tone frequency modulation at a frequency between $10 \mathrm{kHz}$ and $100 \mathrm{kHz}$ on one of the laser was shown to strongly increase the noise floor level as a function of the modulation amplitude. We conclude that this noise floor is due to a down conversion of the residual laser frequency noise, located here mainly between $10 \mathrm{kHz}$ and $150 \mathrm{kHz}$, which is down-converted at low Fourier frequencies by a nonlinearity in the servo loop. Given the sinusoidal shape of the optical frequency discriminator function of the interferometer, the mainly cubic residual nonlinearity around zero can cause such effect. To prove this hypothesis, we have performed numerical simulations to calculate the effect of the nonlinearity of the optical frequency discriminator on the error signal. The results show indeed a white frequency noise with a level in excellent agreement with the experimental measurement. Thanks to the simulation we have been able to derive relations between the residual laser frequency noise and this white noise level. Those relations can be very useful to optimize the choice of the fibre spool length as a function of the frequency noise of the laser to be locked, in order to minimize this noise.

\section{References}

[1] H. Jiang, F. Kéfélian, P. Lemonde, A. Clairon and G. Santarelli, "An agile laser with ultra-low frequency noise and high sweep linearity," Optics Express. 18, 3284-3297 (2010)

[2] C. Salomon, D. Hils and J. Hall, "Laser stabilization at the millihertz level," JOSA B 5, 1576-1587 (1988) 